\title{
A review of visualization techniques of post-mortem computed tomography data for forensic death investigations
}

\author{
Lars Christian Ebert ${ }^{1}$ (i) $\cdot$ Sabine Franckenberg ${ }^{1,2}$ (1) $\cdot$ Till Sieberth $^{1}$ (D) Wolf Schweitzer $^{1} \cdot$ Michael Thali $^{1}$. \\ Jonathan Ford ${ }^{3}$ (1) Summer Decker ${ }^{3}$. S
}

Received: 28 August 2020 / Accepted: 16 March 2021 / Published online: 30 April 2021

(c) The Author(s) 2021

\begin{abstract}
Postmortem computed tomography (PMCT) is a standard image modality used in forensic death investigations. Case- and audience-specific visualizations are vital for identifying relevant findings and communicating them appropriately. Different data types and visualization methods exist in 2D and 3D, and all of these types have specific applications. 2D visualizations are more suited for the radiological assessment of PMCT data because they allow the depiction of subtle details. 3D visualizations are better suited for creating visualizations for medical laypersons, such as state attorneys, because they maintain the anatomical context. Visualizations can be refined by using additional techniques, such as annotation or layering. Specialized methods such as 3D printing and virtual and augmented reality often require data conversion. The resulting data can also be used to combine PMCT data with other 3D data such as crime scene laser scans to create crime scene reconstructions. Knowledge of these techniques is essential for the successful handling of PMCT data in a forensic setting. In this review, we present an overview of current visualization techniques for PMCT.
\end{abstract}

Keywords Postmortem computed tomography $\cdot$ Visualization $\cdot$ Virtual reality $\cdot$ Segmentation $\cdot 3 \mathrm{D}$ printing $\cdot$ Reporting

\section{Introduction}

Forensic cases are increasingly being documented using 3D imaging of both humans (victims, perpetrators) and objects (crime scenes, vehicles, weapons) using a wide variety of scanning techniques [1]. For postmortem imaging, $\mathrm{x}$-ray, computed tomography (CT), CT angiography, magnetic resonance imaging (MRI), and various surface scanning methods are utilized to investigate the circumstances of death of a deceased and to document the findings [2-5]. In such instances, postmortem $\mathrm{CT}$ (PMCT) is the preferred modality; it permits assessment of most forensically relevant conditions such as bone fractures, hemorrhage, parenchymal lacerations, free intracorporal gas accumulation, and the presence of foreign bodies [6, 7]. PMCT

Lars Christian Ebert

lars.ebert@virtopsy.com

1 Zurich Institute of Forensic Medicine, University of Zurich, Zurich, Switzerland

2 Institute of Diagnostic and Interventional Radiology, University Hospital Zurich, Zurich, Switzerland

3 Department of Radiology, University of South Florida, Morsani College of Medicine, Tampa, FL, USA scanning protocols can be optimized for use in forensic cases to increase image quality by using higher energies, thus lowering image noise [2]. In addition, clinical CT scans can be reevaluated forensically for potential use in criminal proceedings. CT scans can also help in 3D documenting objects [8,9].

PMCT generates volumetric data that can be used in a variety of applications in forensic sciences. First, the data can be used as a triage tool for autopsy [10], to thoroughly plan a subsequent autopsy [11] and to assist in determining cause and manner of death [6]. Second, PMCT can be utilized to reconstruct the course of events $[12,13]$, to create comprehensible visualizations for presentation to state attorneys and judges [14], and to add information on the internal body state of the deceased, such as the presence of underlying medical conditions or gunshot trajectories, to 3D-surface scanning [15-17]. This mix of different image modalities adds resolution and color information to the CT scan, which would otherwise be insufficient for the assessment of would surface morphology. Third, it can be used to identify bodies, i.e., in cases of mass disasters [18, 19], thus potentially speeding up disaster victim identification scenarios. Other fields of application include anthropological analyses such as sex or age estimation and identification [20] and medical training [21]. 
Various forensic disciplines, including forensic medicine, forensic anthropology, and forensic crime scene reconstruction, routinely work with postmortem image data $[7,16$, 22]. Forensic radiologists analyze the images for initial diagnosis, and forensic pathologists are able to plan autopsies based on those findings [2]. Both clinical and forensic radiologists primarily use $2 \mathrm{D}$ slices rather than $3 \mathrm{D}$ visualization in the assessment of CT data to perceive subtle details that might otherwise be missed [23]. However, for adequate interpretation of these 2D images of PMCT datasets, precise knowledge of anatomy, awareness of regular postmortem alterations, spatial imagination, and knowledge of the basic operating principles of the examination modality are required. Lacking this type of specialized knowledge, medical laypersons such as prosecutors, lawyers, and judges rely on more comprehensible visualizations of the findings. This information is often provided to them in the form of a printed image report containing annotated 2D images as well as $3 \mathrm{D}$ renderings or dedicated $3 \mathrm{D}$ prints as a supplement $[2$, 24]. In addition, police experts such as forensic officers and technicians can imbed the data and findings from PMCT into their crime scene reconstructions through additional processing steps [15].

Visualization of forensic medical data can be confusing at first due to the large variety of techniques, image modalities, and software available. For every visualization task described in this article, free or open-source software that can be used at no additional cost is available (Table 1). While commercial software products often feature streamlined graphical user interfaces and cutting-edge visualization features to increase their commercial success, they are often quite expensive [25]. In contrast, for those with limited budget, free open-source software may be more affordable and allow independence of vendors, and in some instances, it provides excellent performance [26, 27].
However, free software can come with comparative weaknesses such as insufficient stability, an inferior graphical user interface, and thus a requirement for advanced user skills, lack of support, lack of certification, and lack of accountability, thus ultimately leading to higher costs [28].

In this review article, we introduce different methods of visualizing PMCT in 2D and 3D and give examples on how to use these techniques, based on the current scientific literature. We show how to convert PMCT data to polygon models that are suitable for 3D printing, metrology, and $3 \mathrm{D}$ reconstruction. We limit this review to techniques that are available in widely used software. We also provide a list of open-source and free software tools to give a starting point for the visualization of PMCT data. The review is structured in two main components: $2 \mathrm{D}$ and $3 \mathrm{D}$ visualizations. First, for the 2D section, data types used in conjunction with visualizing PMCT data are introduced. This is followed by 2D visualization techniques, as they are often used for analyzing PMCT images radiologically. For the 3D section, 3D visualization methods are discussed, following a workflow that is often used when visualizing in 3D - first, direct volume visualization using volume rendering, followed by the generation of polygon models through segmentation for specialized visualization applications. Finally, different visualization options for polygonal data such as 3D printing or virtual reality/ augmented reality are introduced.

\section{Visualization techniques}

\section{Data types}

The Digital Imaging and Communication in Medicine (DICOM) standard is the standard format for storing
Table 1 List of free and opensource software that can be used in different visualization tasks

\begin{tabular}{lll}
\hline Task & Software & Website \\
\hline 2D visualization & Horos & https://horosproject.org/ \\
2D annotation & Gimp & https://www.gimp.org/ \\
2D Bitmap editing/layering & Inkscape & https://inkscape.org/ \\
2D annotation & LibreOffice Impress & https://www.libreoffice.org/ \\
& Horos & https://horosproject.org/ \\
3D VRT & 3D Slicer & https://www.slicer.org/ \\
& MeVisLab & https://www.mevislab.de/ \\
3D VRT, Physical Shader & 3D Slicer & https://www.slicer.org/ \\
Segmentation & MeshLab & http://www.meshlab.net/ \\
3D polygon processing & Blender & https://www.blender.org/ \\
3D polygon rendering & Unity & https://unity.com/ \\
Virtual reality & Gimp & https://www.gimp.org/ \\
2D Bitmap editing & Slic3r & https://slic3r.org/ \\
3D printing & &
\end{tabular}




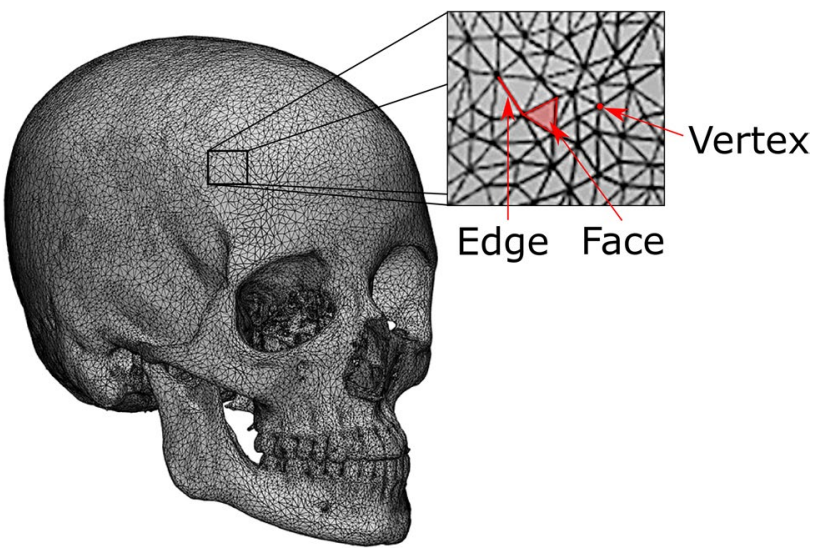

Fig. 1 Polygon model describing the surface of an anthropologist's skull, based on data extracted from a CT scan. Polygon meshes consist of points called vertexes that are interconnected by their edges to form polygons or faces

digital medical images [29]. An image stored in DICOM format consists of two parts-the image data and a "header" containing information such as patient data and the scanning protocol used. $3 \mathrm{D}$ volumetric data such as $\mathrm{CT}$ scans are stored in stacks of images contained in separate DICOM files. A CT volume consists of single elements called volume picture elements (voxels), which are similar to picture elements (pixels) in a 2D image. Each voxel has a position in the volume and a value depending on the image modality; in the case of CT data, the value is the $\mathrm{x}$-ray attenuation measured in Hounsfield units (HU; for more detailed information, see the following section) [30].

While voxels are the standard for visualizing medical images, many software packages, such as the ones used in crime scene reconstruction or 3D printing, require polygon meshes. Polygon meshes consist of 3D points called vertexes that are interconnected through edges to form polygons (Fig. 1). While volumetric data consisting of voxels describe the entire volume of an object, including its insides, polygon meshes are used to describe the shape of an object [31]. Using segmentation, volume data can be converted into polygon meshes.

\section{D visualization}

HU are a measure of $\mathrm{x}$-ray attenuation at a specific location, with increasing values signifying more attenuation [32]. The Hounsfield scale ranges from -1000 for gases to 0 for water to several thousand HU for metals. HU are usually stored using 12 bits per voxel, which means that every voxel can have one of 4096 different values. Because standard computer screens are only able to display a limited number of shades of gray (typically 256), CT datasets cannot be displayed directly, and further processing is required. Typically, this is done by windowing [33]. A range (the window) of HU that most likely contains the structures of interest is selected and mapped to the 256 available grayscale values. All voxels with values below the lower limit of the selected window are displayed as black, and all voxels with values above its upper limit are displayed as white. A window consists of two values, a defined position on the HU histogram and a width. Some structures and pathologies are only perceivable in a specific window. An example of displays obtained using different window settings and the same dataset can be seen in Fig. 2.

\section{Multiplanar and curved reformations}

To improve the assessment of certain anatomical regions and pathologies, acquired data, mostly from the axial plane, can be reformatted to nonaxial planes in a so-called multiplanar reformation (MPR) (Fig. 3). For an MPR, the 3D volume is resliced into planes that can be arbitrarily tilted
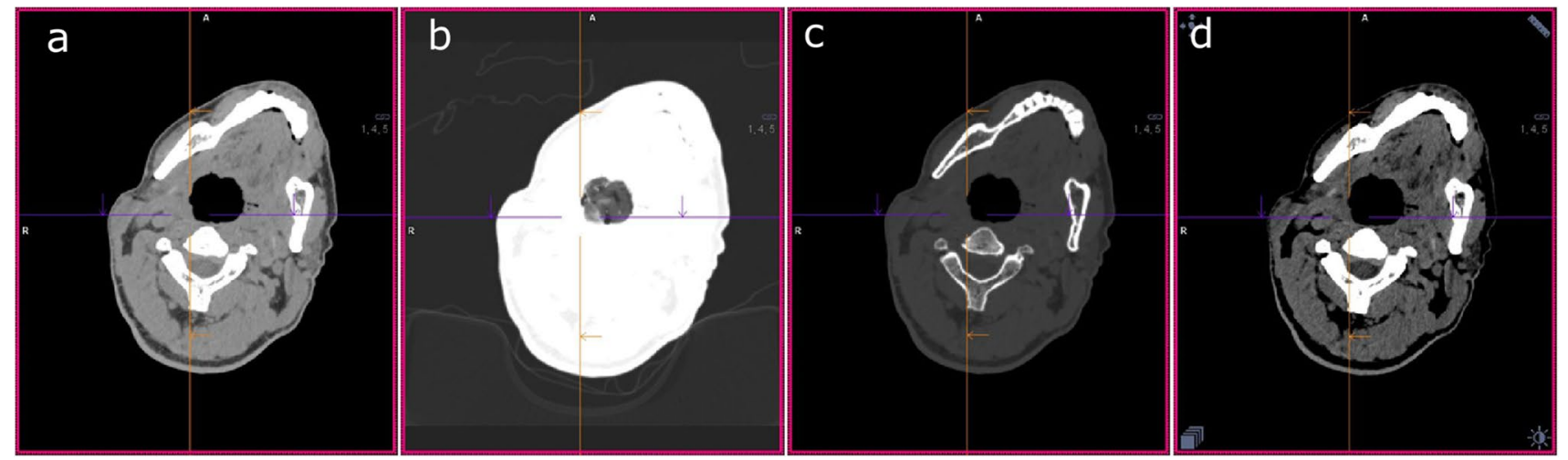

Fig. 2 PMCT scan of the neck, visualized in different windows. (a) soft tissue window; (b) lung window; (c) bone window; (d) brain window. Note that the material obstructing the airways is only visible in image b, the lung window, and does not appear in any of the other images 
and positioned. To avoid stairstep artifacts, the scan resolution must be sufficiently high in all directions [34]. MPR is one of the basic techniques that is used to assess CT images and to visualize findings in forensic radiology; among other applications, it can be used in the reconstruction of gunshot wounds [35], the visualization of foreign bodies [36], and victim identification [37]. As a subcategory of MPR, curved MPR is also available. In curved MPR, instead of reformatting the $\mathrm{CT}$ data along a chosen plane, a 2D image is generated along a selected curve (e.g., such as a coronary artery), which results in clearer depictions of certain anatomical structures and pathologies that would be difficult to comprehend when viewed in a plane. In the forensic context, MPR is, for example, used in the visualization of the entire skull in one image [38] (Fig. 4), in the visualization of the whole rib cage to assess rib fractures [39] and in the creation of a virtual orthopantomogram by following the contours of the dental arches [40].

\section{Maximum and minimum intensity projections (MIP, MinIP)}

MIP and MinIP are two additional visualization methods that use the data in a defined volume to generate a 2D image. In MIP, the voxel with the highest attenuation value on every slice throughout the selected volume is projected onto a $2 \mathrm{D}$ image [41], which means that MIP emphasizes high-density materials such as bone and metal. This method can be used in the localization and determination of foreign bodies, for example, in identification based on the presence of dental restorative materials [2, 42, 43]. In contrast, in MinIP, the voxel with the lowest attenuation value on every slice throughout the selected volume is projected onto a $2 \mathrm{D}$ image [44], thereby emphasizing low-density structures such as gases. This method contributes, for example, to the visualization of gas embolism, emphysema, or decompositional gas [45, 46] (Fig. 5). MinIP can also be used to evaluate other structures with large differences in attenuation, such as in the display of fluid-filled bronchi in cases of drowning [47].

In addition to MIP and MinIP, when projecting the average attenuation of the voxels throughout the selected volume, a conventional radiograph is approximated that can be used in the creation of a virtual orthopantomogram from PMCT data [48].

\section{Volume visualizations}

Frequently, 2D visualizations may be supplemented by 3D visualizations. An early technique allowing for such a 3D visualization was surface-shaded displays (SSD); this technique only visualizes voxels within a defined Hounsfield range, uses a simplistic lighting model, and allows realtime rendering. SSD have been mostly replaced by volumerendering techniques (VRT) in most medical image viewers due to the increasing processing power available. Similar to windowing in $2 \mathrm{D}$ images, in which $\mathrm{HU}$ are mapped to grayscale values, in VRT a voxel is rendered by mapping HU to color and opacity values [41]. This mapping is called a transfer function. By defining appropriate transfer functions, specific structures can be highlighted or diminished in the final presentation (Fig. 6). Furthermore, a variety of tools, such as clipping planes and punch tools, allow the user to remove parts of the volume dataset to achieve a better depiction of the desired finding.

Volume rendering can create a simplified pictorial representation of significant findings in 3D [49]. Yet, while visually pleasing, volume-rendered models alone are often too imprecise for use in the assessment of CT data [23]. When used as a supplement to $2 \mathrm{D}$ visualizations, they enable a quick overview (i.e., fracture patterns, foreign bodies) in the initial analysis process, a capability that is specifically useful in high-resolution datasets [23]. Furthermore, they are visually more accessible to medical laypersons (i.e., for
Fig. 3 MPR of a suicidal gunshot injury with the entry wound on the right and the exit wound on the left side. The reconstruction follows the path of the bullet through the brain. (a) coronal view; (b) axial view

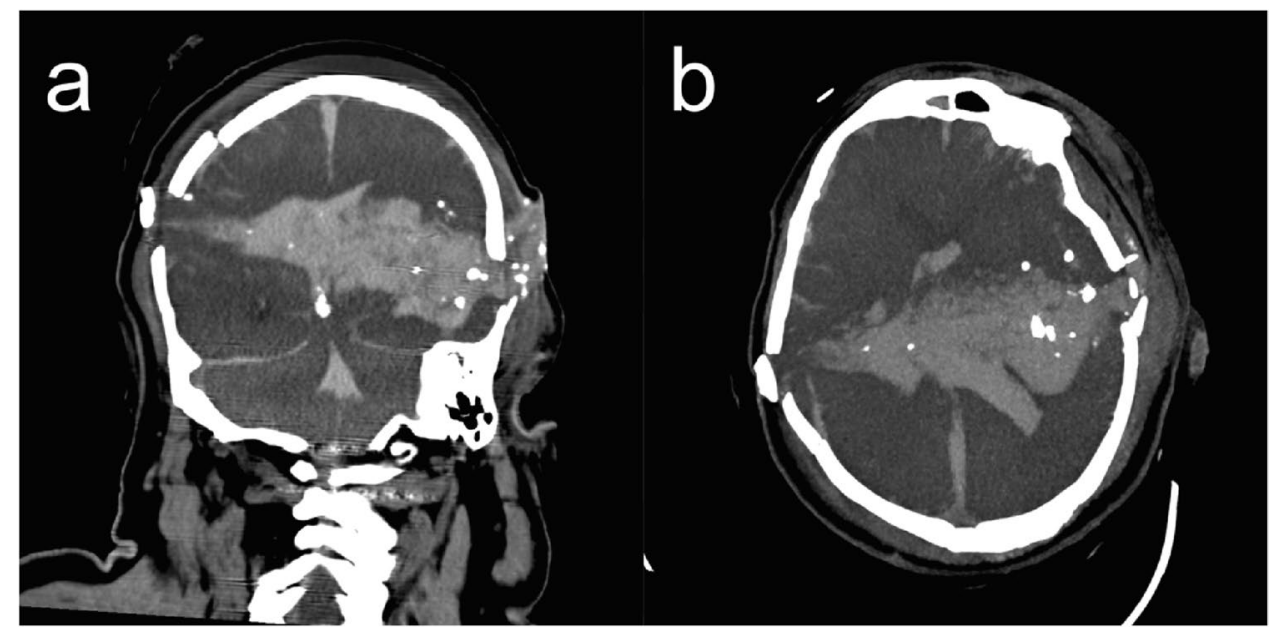




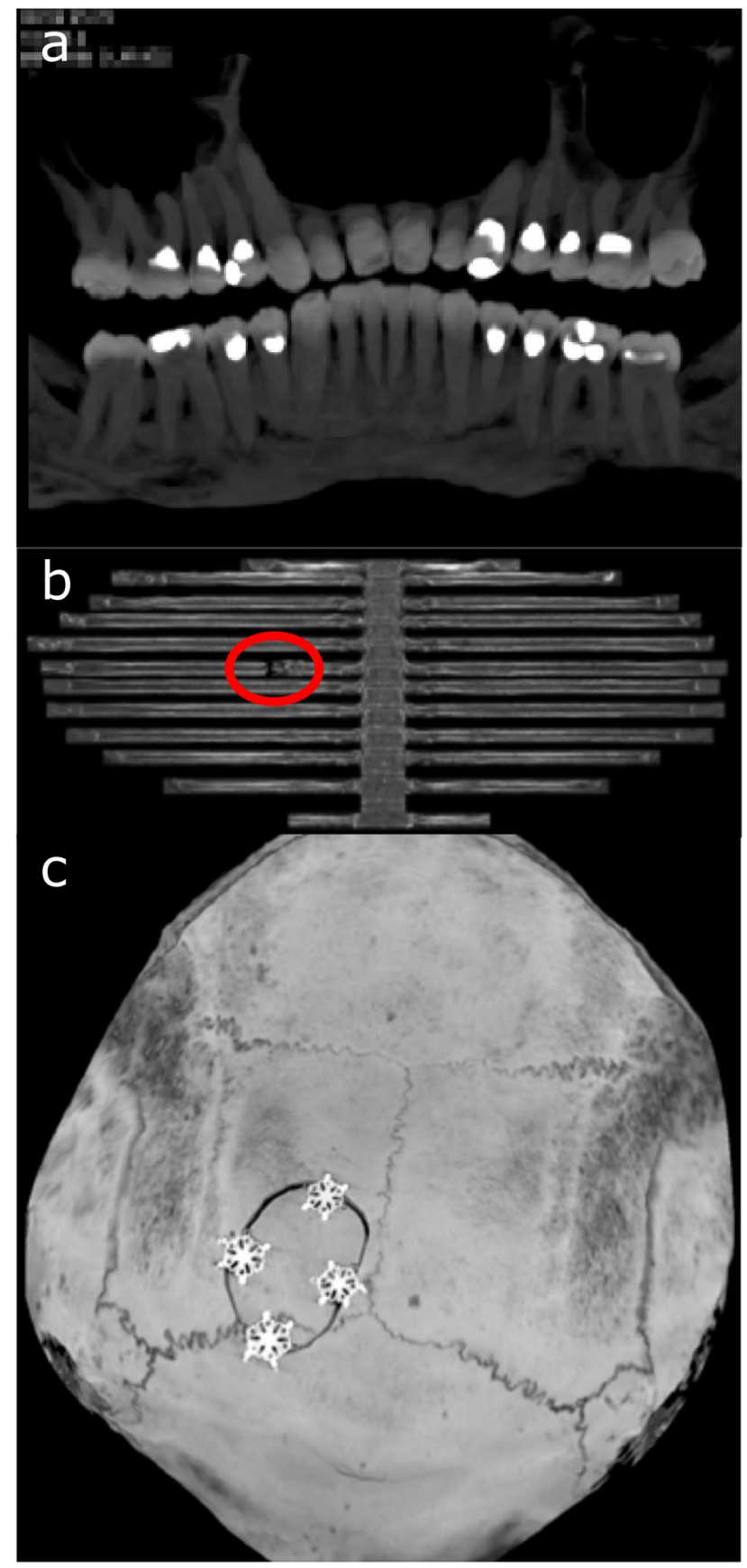

Fig. 4 Different types of complex 2D reconstructions. a Curved MPR of an individual's dentition, simulating an orthopantomogram that can be compared to antemortem images. b Rib unfolding that can be used in the rapid assessment of multiple rib fractures (c). Skull unfolding showing surgical plates and screws after surgical intervention

presentation in a courtroom) and are considered the preferred visualization method by state attorneys [24, 31].

More recently, new sophisticated rendering algorithms even permit the simulation of photon behavior, resulting in the correct calculation of optical effects such as depth-of-field and shadowing. These techniques promise more realistic visualization and better depth perception, but this is achieved at the expense of rendering speed [50] (Fig. 7). In general, low-resolution data or data containing image artifacts will result in insufficient visualizations, especially in 3D [34].

Visualization, specifically when 3D rendering is used, also holds certain dangers in forensics. Realistic-looking images and models will be more readily accepted as valid without further questions, as evidenced by Siemens Healthineers release of Cinematic Rendering. This phenomenon of "if it looks plausible, it is plausible" is called naïve realism $[51,52]$ and should always be kept in mind when creating visualizations to avoid undue influence or prejudice.

\section{Segmentation}

Segmentation is the principal tool used to generate 3D polygon models of specific anatomical structures (such as a whole organ) from 2D CT slices. Using various techniques, each voxel is assigned a label that allows the differentiation of tissues, organs, and anatomical structures. Depending on the method (manual, semiautomatic, or automatic), the accuracy of this tool is to a certain degree influenced by the experience of the operator [53]. Successful use of segmentation requires expert knowledge of anatomy and pathology as well as knowledge of the available tools. Lack of expertise might introduce serious errors in the segmented volume and in the subsequent assessment. In addition, all visualization techniques are highly dependent on the quality and resolution of the data [54]. Another disadvantage of manual segmentation is that it is a rather time-consuming and labor-intensive process. Semiautomatic methods such as region growing [55], which automatically selects all voxels within a defined Hounsfield range that are connected to a seed point specified by the operator, are used to speed up the segmentation process. Still, even fully automatic segmentation methods require some sort of external influence by the operator, such as in the selection of initial parameters, and this significantly affects the model outcome [56].

Usually, a combination of different segmentation tools and methods is used to perform a segmentation task. The most common methods are thresholding, region growing, livewire, classifiers, clustering, machine learning, and atlasguided approaches [57-59]. Examples of segmented and unsegmented datasets can be seen in Fig. 8.

In thresholding, a defined range of attenuation values (HU) is identified in all pixels to highlight a desired structure. Thresholding is primarily used in the visualization of findings with a high attenuation difference from the surrounding tissue, such as bones, contrast agents, or metallic foreign bodies. Thresholding is of 
Fig. 5 Different volume visualizations of the same PMCT dataset. a Averaging visually mimics the appearance of a plain radiograph. b MIP highlighting structures with high $\mathrm{x}$-ray density such as bone. The high-density material visible in this image is debris that is located underneath the body. c MinIP visualizing the gas distribution inside the body and excluding the air surrounding the body

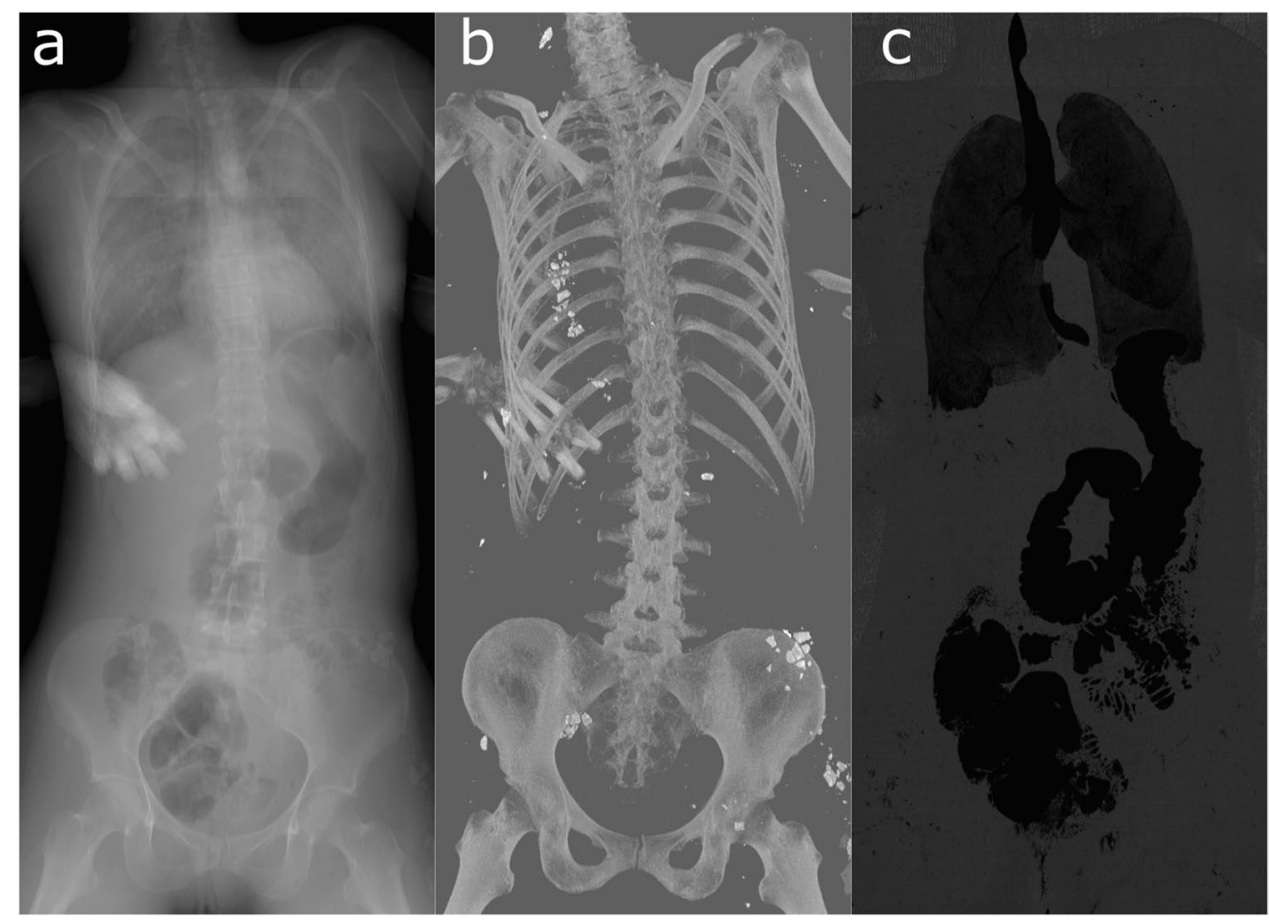

limited use in the segmentation of parenchymal organs and soft tissue lesions due to their small differences in attenuation values. The operator must define the range of attenuation values individually accordingly to the structures that are to be imaged.

Region growing is often used to further identify a desired structure that is predefined by an initial other segmentation method (mostly thresholding). Region growing checks for interconnectivity using the predefined parameters and then isolates an object of interest. Region growing can also be dynamic where a seed point is selected by the modeler. The segmentation expands from the seed point to the neighboring pixels based on the user's criteria, such as intensity or a gradient. The segmentation process continues to expand until the neighboring pixels no longer satisfy the initial criteria [56].

Livewire segmentation is one type of semiautomatic method in which the user clicks on the object of interest and draws a delineation around it [60], which can be done along any of the two planes of view and will automatically segment the third plane. This method can speed up the process of segmenting structures by using a contour that is visible to the operator.

Classifiers rely on pattern recognition to isolate structures in a feature space. They require training data from manually segmented sources to use as a reference for

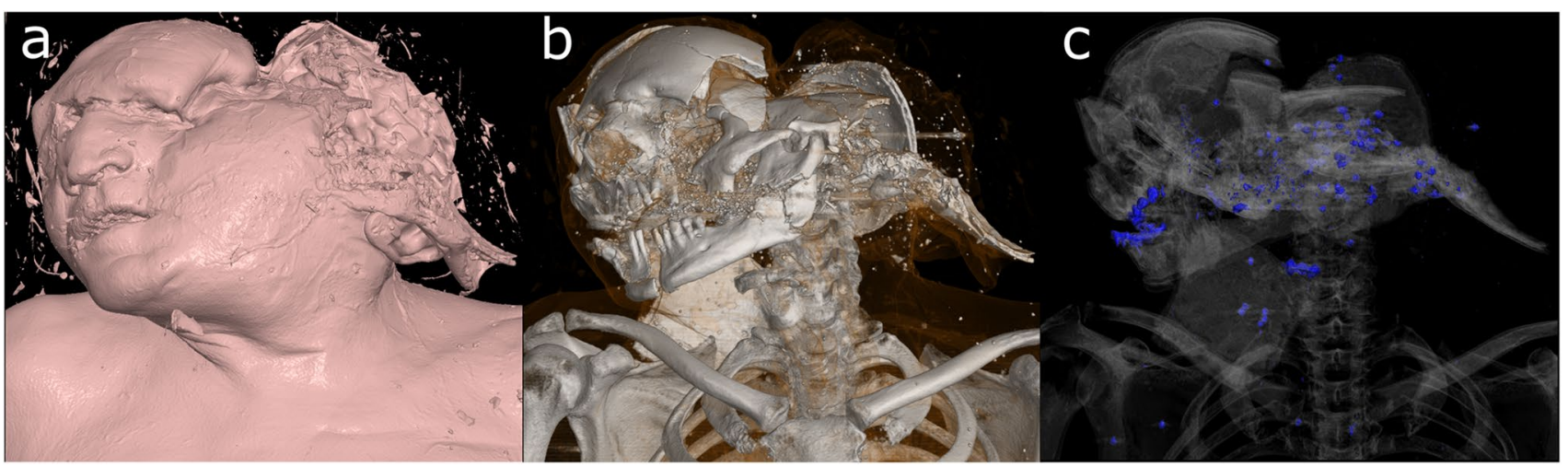

Fig. 6 VRT of a suicidal shotgun injury demonstrating visualization using different transfer functions. (a) Surface visualization. (b) Bone visualization. (c) Visualization of radio dense material in blue 
Fig. 7 Cinematic rendering of a suicidal gunshot injury to the head. The rendering demonstrates increased perceived realism through the physically correct calculation of optical effects such as shadowing. a View from the left showing the exit wound. b Frontal view with skull fracture. c View from the right with entry wound

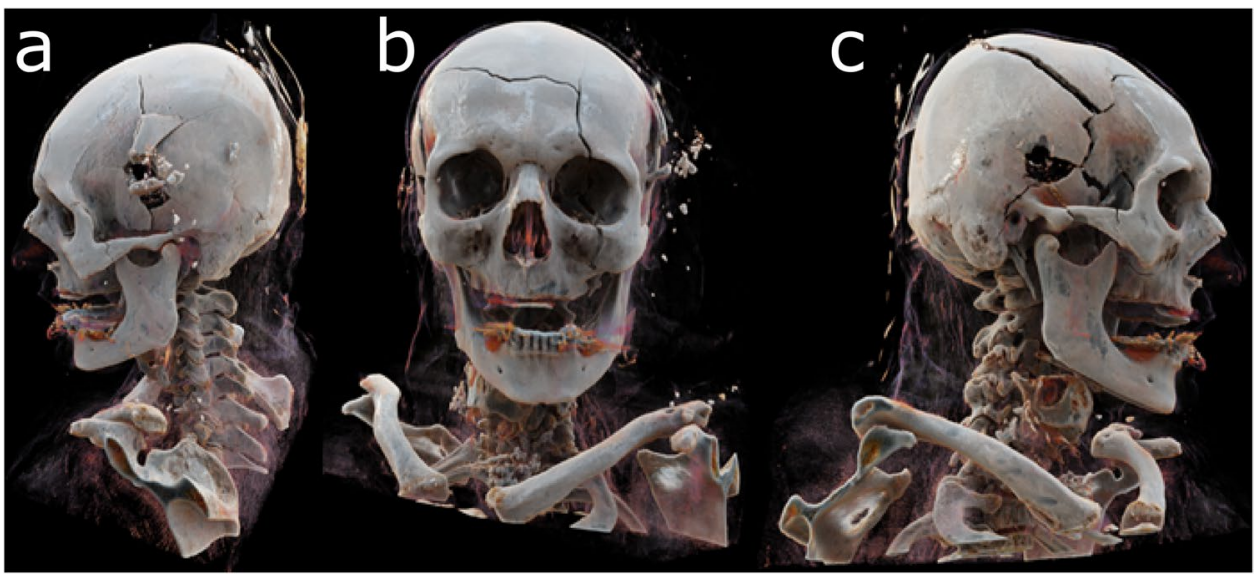

automatically segmenting new data. The creation of these training sets relies on manual modeling and therefore is subject to observer error. Clustering is very similar to classifier methods but does not utilize training data. Clustering does, however, require initial input from direct segmentation, but it does not distinguish well between regions of interest and is sensitive to noise and image inhomogeneities [57].

More advanced methods of image segmentation include machine learning approaches, atlas-based approaches, or statistical shape modeling (SSM), all of which aim towards the ultimate goal of fully automatic segmentation of features or structures [61-65].

Regardless of the method used, proper segmentation is vital because it is the basis of all resulting 3D data and models. Any resulting work is only as good as the initial imaging capture and segmentation.

\section{Polygon visualization}

Segmentation masks (labeled voxels) can be converted to polygon meshes using techniques such as the marching cubes algorithm [66]. The resulting 3D surface models generated from CT data are only as good as the resolution quality of the image data. Thicker slices may result in stepped or pixellatedappearing models [34]. The surface models are representations of the surface of the actual physical object and are the base format for methods such as morphometrics [67], virtual physics simulation [68], 3D printing [69], and enhanced visualization or animation [16]

Once a 3D model is generated, it can be exported from the software package as an STL, an OBJ, or in another appropriate file format. In all 3D modeling methods, once a 3D mesh is generated, it is rarely left in its initial capture state, and additional processing steps are required. Any holes in the
Fig. 8 Segmented data. a Sagittal view of a PMCT dataset. b Segmented spine. Each vertebra, disc, and the sacrum has been semiautomatically segmented and color-coded

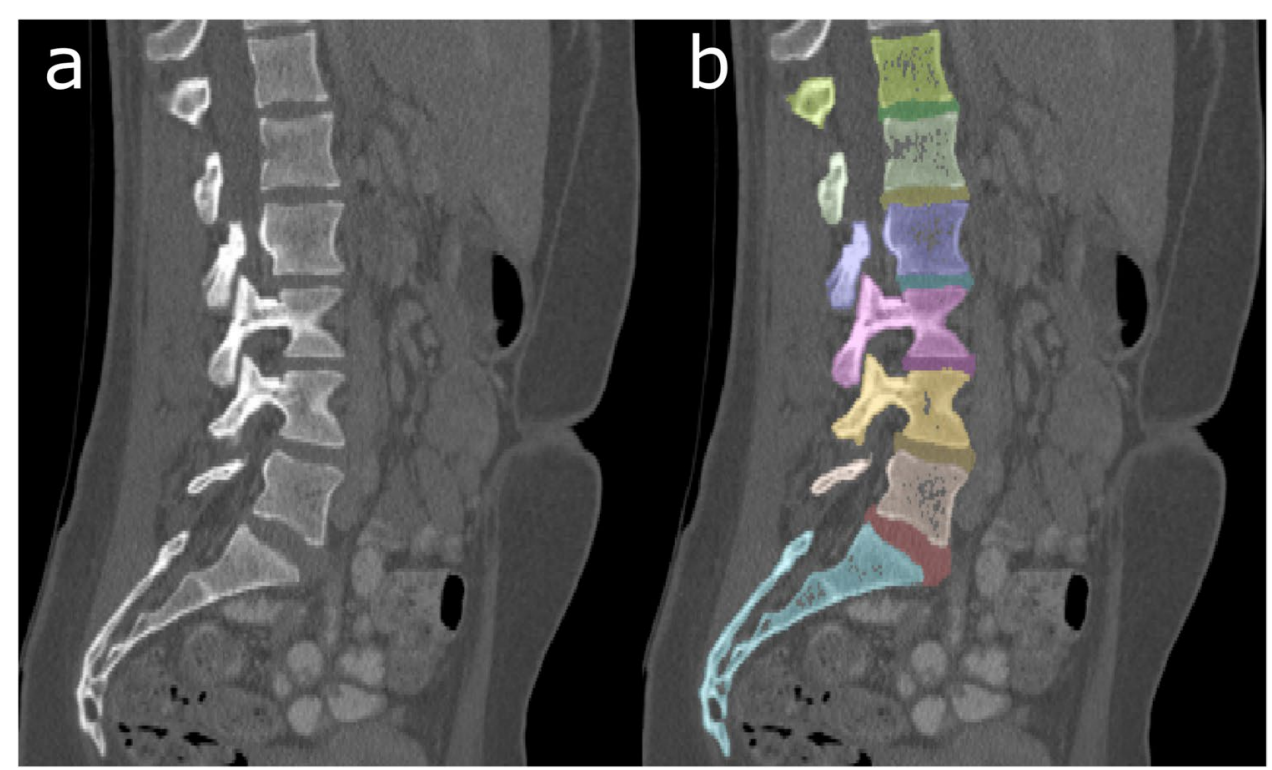


mesh must be patched or filled for it to run properly in other software. The number of triangles of a mesh must be reduced for ease of computation. Various algorithms can check and repair a 3D mesh to remove potential issues such as intersecting or overlapping triangles or poor triangle quality. Artifacts of the scanning process also need to be removed. Noise reduction will be handled through global, local, or manual smoothing methods. Noise shells, stray bits of mesh that do not contribute to the object, need to be removed. It is possible to convert 3D polygon meshes into 3D PDFs that can be viewed using standard software [70], which offers a means to provide $3 \mathrm{D}$ data to prosecutors and judges.

\section{Mixing and layering}

Each visualization method has its respective advantages and disadvantages. It is, however, possible to employ multiple methods to achieve the desired result. For example, polygon rendering and VRT can be used in conjunction (Fig. 9). It is also possible to incorporate additional information. As an example, small CT-dense particles may be projected onto 3D surfaces and combined with an SSD or VRT reconstruction to allow better visual recognition in combination with

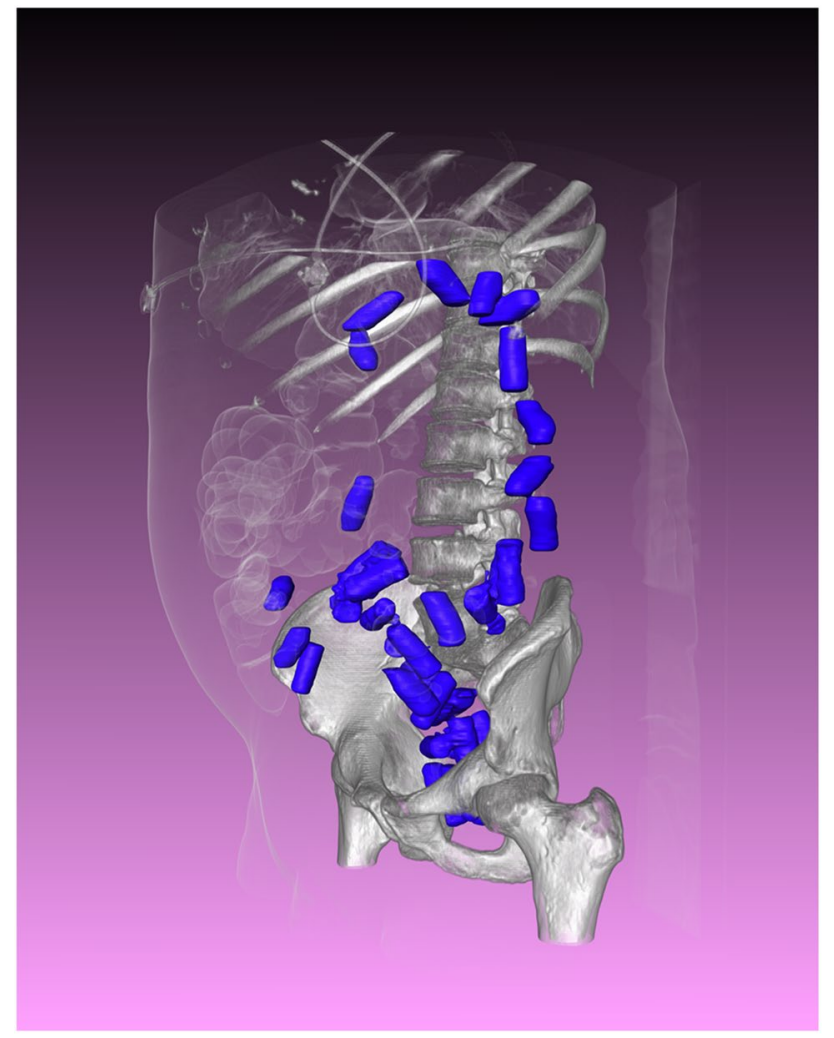

Fig. 9 Visualization of drug containers on PMCT in a case of body backing. VRT of soft tissue and bone was mixed with a polygon rendering resulting from segmentation of the drug containers anatomical references [71]. An alternative to mixing different visualizations is layering of multiple VRTs that have been rendered using different transfer functions (Fig. 10).

\section{Annotations and highlighting of image reports}

Especially for medical laypersons, 2D slides extracted from cross-sectional imaging datasets may be difficult to understand. The anatomical context is often not clear, and the lack of actual colors can add to the problem. Proper annotation of 2D images, including color coding, markers, and arrows, is usually not available in medical visualization software but can be added later using software such as LibreOffice Impress or Inkscape (Table 1). In 3D visualizations, annotations can help localize specific findings (Fig. 11).

\section{D printing}

3D printing is a rapid prototyping technique that allows the physical creation of an object based on digital information (Fig. 12). A replica of any object that has been captured in 3D may be produced via 3D printing. 3D printing makes it possible to analyze and present objects in a way that originally could only be accomplished via destructive means [72]. The ability to create duplicate 3D structures

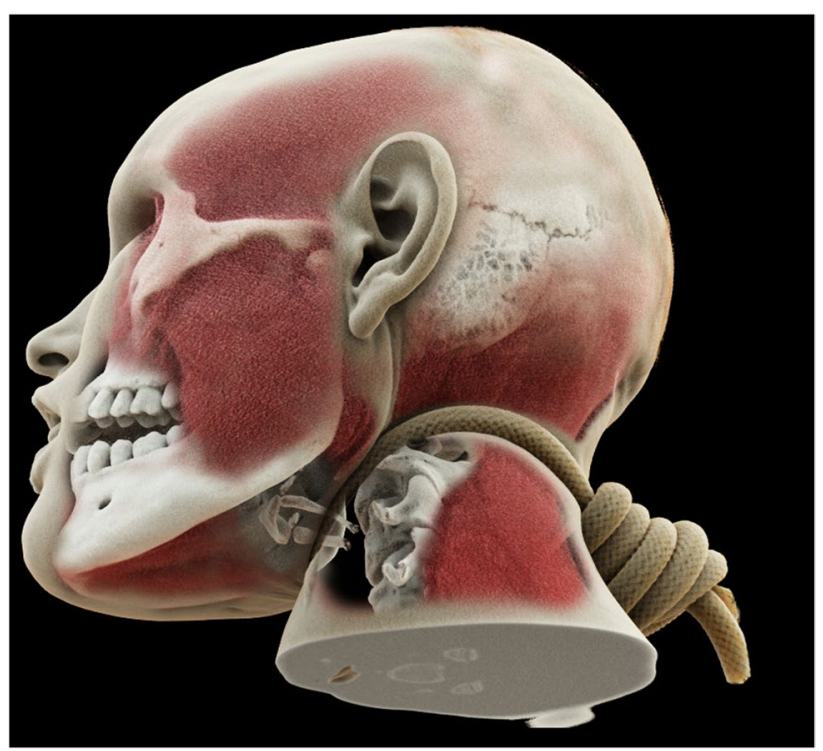

Fig. 10 Visualization of a hanging using multiple layered cinematic renderings (bone, muscle body, and surface). Using this method, the bony structures of the neck and the rope can be visualized at the same time despite the fact that the rope has a Hounsfield density similar to that of the soft tissue. Painted transparencies on different layers allow exposure of underlying structures such as the hyoid bone 

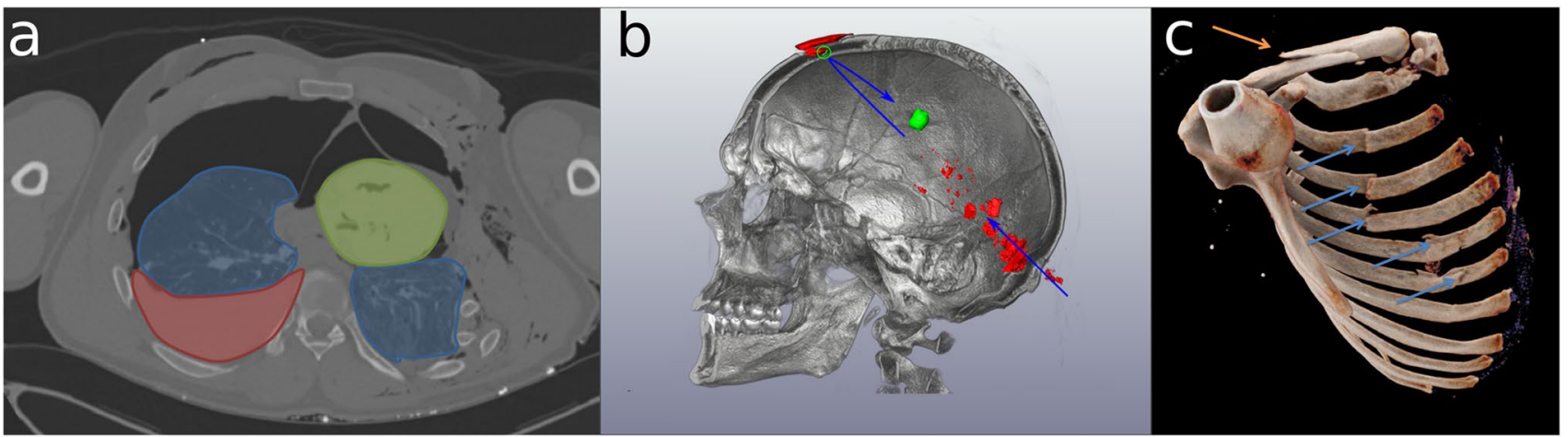

Fig. 11 Annotated images. a Visualization of hemothorax and tension pneumothorax by color-coding of different structures. Heart (green), lungs (blue), and blood (red). b Combined VRT and polygon rendering displaying dislodged bone fragments. Additional annotations

in another material allows the creation of artificial replicas of funerary and forensic remains. The application of this technology is limited only by its availability, by the user's ideas, and, of course, by the technical limitations of the software and 3D printing devices used. 3D printing today has gone beyond making device prototypes. Many printers will print to the micron level, making them valuable to forensic practitioners [73] and even in clinical applications for diagnostic and surgical planning purposes.

There are countless professional and hobbyist 3D printers on the market. Each printer uses either some sort of fused deposition modeling (FDM), stereolithography (SLA), selective laser sintering (SLS), selective laser melting (SLM), or electronic beam melting (EBM). FDM uses a variety of materials, the most common being PLA or ABS plastics [27]. To create the print, it heats the thermoplastic material so that it will flow and then builds an

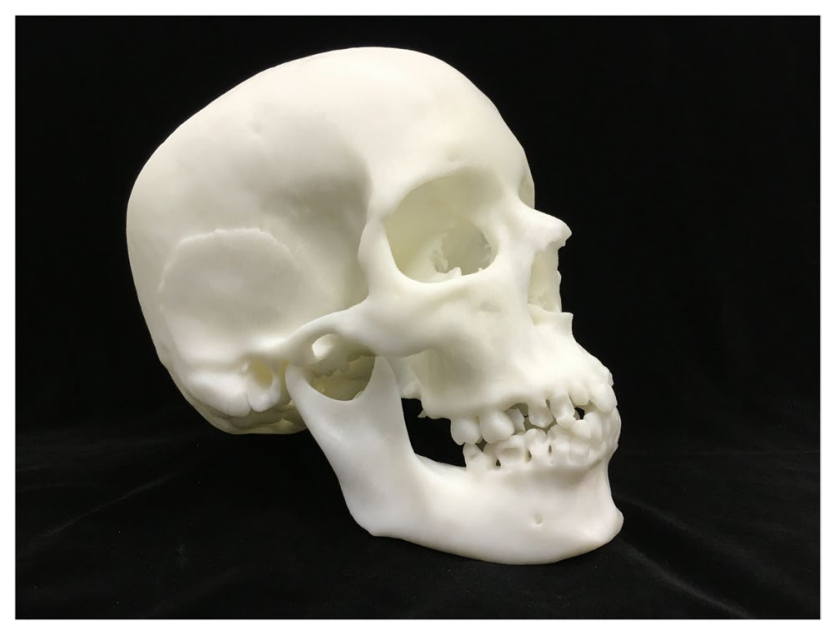

Fig. 12 3D-printed skull extracted from a CT scan using FDM printing highlight the bullet path as well as a smaller projectile fragment. c Cinematic rendering of fractures of the ribs and clavicular bone, annotated using different-colored arrows

object layer by layer. Figure 8 shows an image of a skull that was printed using FDM.

SLA technology uses a liquid resin that interacts with light/ lasers to solidify each layer to create the desired object. SLS uses a laser to heat powdered metal so that it fuses with its neighbors to form the desired shape. SLM is similar to SLS, but instead of heating the metal to the point of fusion, SLM melts it so that it combines with its neighbor. EBM uses similar technology, but instead of lasers, it uses an electron beam. The types of materials utilized in these 3D printers include plastics, resins, glass, ceramics, and metals. $3 \mathrm{D}$ printing is not cheap, but there are affordable desktop varieties available such as MakerBot, FormLabs, and 3D Systems. The larger and more expensive SLS machines are usually limited to large industrial manufacturers. However, outsourcing of 3D printing to commercial facilities is always an option if in-house printing is not feasible, taking into account data privacy.

The forensic community uses 3D printing technology in various applications [74]. This includes forensic facial reconstruction [75], weapon matching [76], presentation of evidence in court $[69,77]$, anthropological evidence reconstruction $[78,79]$, and educational purposes in a classroom setting [80]. As an additional application, 3D printing can be used to quickly manufacture workpart prototypes (rapid prototyping) [81]. Rapid prototyping has relevance in forensics because if allows for the development of custom made parts in low quantities [82].

\section{Stereoscopic displays/AR/VR}

Conventionally, medical imaging data, even when rendered in $3 \mathrm{D}$, are usually displayed as $2 \mathrm{D}$ projections and displayed on a screen. This means that scale and depth information is lost on such renderings. Stereoscopic displays that offer a real 3D impression have shown to have advantages in clinical settings 
such as pre-operative planning or minimally invasive surgery. They can also help in understanding complex 3D structures [83]. When positional and rotational tracking of the users head is added to 3D displays, virtual reality (VR) and augmented reality (AR) applications are possible. While virtual reality creates a scene independent of the physical surroundings, augmented reality provides additional information that is embedded in the real world surrounding the viewer. Visualizing using AR means that the embedding of information can provide a forensic expert with additional information during autopsy, for example, by providing access to image data [84, 85]. In theory, both VR and AR can display volumetric and polygonal data in 3D. However, AR devices usually do not have the processing capabilities required for VRT. Some of the software packages used for medical visualization (such as $3 D$ Slicer) offer VR support through plugins [86]. Meshes that have been extracted from medical datasets can be incorporated into incident reconstructions that can be visualized in VR for various purposes such as displaying the reconstruction to state attorneys or conducting reconstructions or virtual crime scene visits [87-89].

\section{Conclusion}

PMCT is an image modality that is often used in forensic medicine and forensic incident reconstructions. Case- and audience-specific visualization is vital to identifying relevant findings and communicating them appropriately. A large number of different 2D and 3D techniques, each of which has specific applications, exist. Knowledge of these techniques is essential to successfully handling PMCT data in a forensic setting.

Funding Open Access funding provided by Universität Zürich.

Data availability Not applicable.

Code availability Not applicable.

\section{Declarations}

Ethics approval Ethical approval for using anonymized post-mortem image data in research has been waived by the Ethical Committee of the Canton of Zurich (KEK-ZH No: 2015-0686).

Conflict of interest The authors declare no competing interests.

Open Access This article is licensed under a Creative Commons Attribution 4.0 International License, which permits use, sharing, adaptation, distribution and reproduction in any medium or format, as long as you give appropriate credit to the original author(s) and the source, provide a link to the Creative Commons licence, and indicate if changes were made. The images or other third party material in this article are included in the article's Creative Commons licence, unless indicated otherwise in a credit line to the material. If material is not included in the article's Creative Commons licence and your intended use is not permitted by statutory regulation or exceeds the permitted use, you will need to obtain permission directly from the copyright holder. To view a copy of this licence, visit http://creativecommons.org/licenses/by/4.0/.

\section{References}

1. Carew RM, Errickson D (2019) Imaging in forensic science: five years on. J Forensic Radiol Imaging 16:24-33. https://doi.org/10. 1016/j.jofri.2019.01.002

2. Flach PM, Gascho D, Schweitzer W et al (2014) Imaging in forensic radiology: an illustrated guide for postmortem computed tomography technique and protocols. Forensic Sci Med Pathol 10:583-606. https://doi.org/10.1007/s12024-014-9555-6

3. Grabherr S, Grimm J, Dominguez A et al (2014) Advances in post-mortem CT-angiography. Br J Radiol 87:20130488. https:// doi.org/10.1259/bjr.20130488

4. Jalalzadeh H, Giannakopoulos GF, Berger FH et al (2015) Postmortem imaging compared with autopsy in trauma victims - a systematic review. Forensic Sci Int 257:29-48. https://doi.org/10. 1016/j.forsciint.2015.07.026

5. Ebert LC, Flach P, Schweitzer W et al (2016) Forensic 3D surface documentation at the Institute of forensic medicine in Zurich workflow and communication pipeline. J Forensic Radiol Imaging 5:1-7. https://doi.org/10.1016/j.jofri.2015.11.007

6. Ampanozi G, Halbheer D, Ebert LC et al (2020) Postmortem imaging findings and cause of death determination compared with autopsy: a systematic review of diagnostic test accuracy and meta-analysis. Int J Legal Med 134:321-337. https://doi.org/10. 1007/s00414-019-02140-y

7. Norberti N, Tonelli P, Giaconi C et al (2019) State of the art in post-mortem computed tomography: a review of current literature. Virchows Arch 475:139-150. https://doi.org/10.1007/ s00428-019-02562-4

8. Inokuchi G, Yoshida M, Makino Y, Iwase H (2019) Utility of contrast-enhanced computed tomography in forensic examination of a stab wound in living individuals. Forensic Sci Med Pathol 15:463-469. https://doi.org/10.1007/s12024-019-00133-2

9. Matusz EC, Schaffer JT, Bachmeier BA et al (2020) Evaluation of nonfatal strangulation in alert adults. Ann Emerg Med 75:329338. https://doi.org/10.1016/j.annemergmed.2019.07.018

10. Chatzaraki V, Heimer J, Thali M et al (2018) Role of PMCT as a triage tool between external inspection and full autopsy - case series and review. J Forensic Radiol Imaging 15:26-38. https:// doi.org/10.1016/j.jofri.2018.10.002

11. Ezawa H, Shiotani S, Uchigasaki S (2007) Autopsy imaging in Japan. Rechtsmedizin 17:19-20. https://doi.org/10.1007/ s00194-006-0409-8

12. Ebner L, Flach PM, Schumann K et al (2014) The tip of the tip of the knife: stab sequence reconstruction using postmortem CT in a homicide case. J Forensic Radiol Imaging 2:205-209. https:// doi.org/10.1016/j.jofri.2014.08.002

13. Flach PM, Ampanozi G, Germerott T et al (2013) Shot sequence detection aided by postmortem computed tomography in a case of homicide. J Forensic Radiol Imaging 1:68-72. https://doi.org/ 10.1016/j.jofri.2013.03.045

14. Schweitzer W, Bartsch C, Ruder TD, Thali MJ (2014) Virtopsy approach: structured reporting versus free reporting for PMCT findings. J Forensic Radiol Imaging 2:28-33. https://doi.org/10. 1016/j.jofri.2013.12.002 
15. Buck U, Naether S, Braun M et al (2007) Application of 3D documentation and geometric reconstruction methods in traffic accident analysis: with high resolution surface scanning, radiological MSCT/MRI scanning and real data based animation. Forensic Sci Int 170:20-28. https://doi.org/10.1016/j.forsciint.2006.08.024

16. Buck U, Naether S, Räss B et al (2013) Accident or homicide virtual crime scene reconstruction using 3D methods. Forensic Sci Int 225:75-84. https://doi.org/10.1016/j.forsciint.2012.05.015

17. Villa C, Olsen KB, Hansen SH (2017) Virtual animation of victim-specific 3D models obtained from $\mathrm{CT}$ scans for forensic reconstructions: living and dead subjects. Forensic Sci Int 278:e27-e33. https://doi.org/10.1016/j.forsciint.2017.06.033

18. Reichs KJ, Dorion RBJ (1992) The use of computed tomography (CT) scans in the comparison of frontal sinus configurations. $J$ Can Soc Forensic Sci 25:1-16. https://doi.org/10.1080/00085030. 1992.10756997

19. Ruder TD, Kraehenbuehl M, Gotsmy WF et al (2012) Radiologic identification of disaster victims: a simple and reliable method using CT of the paranasal sinuses. Eur J Radiol 81:e132-138. https://doi.org/10.1016/j.ejrad.2011.01.060

20. Decker SJ, Davy-Jow SL, Ford JM, Hilbelink DR (2011) Virtual determination of sex: metric and nonmetric traits of the adult pelvis from $3 \mathrm{~d}$ computed tomography models*, $\dagger$. J Forensic Sci 56:1107-1114. https://doi.org/10.1111/j.1556-4029.2011. 01803.x

21. Buenting M, Mueller T, Raupach T et al (2016) Post mortem CT scans as a supplementary teaching method in gross anatomy. Ann Anat - Anat Anzeiger 208:165-169. https://doi.org/10.1016/j. aanat.2016.05.003

22. Christensen A, Smith M, Gleiber D et al (2018) The use of X-ray computed tomography technologies in forensic anthropology. FA 1:124-140. https://doi.org/10.5744/fa.2018.00132018.0013

23. Andriole KP, Wolfe JM, Khorasani R et al (2011) Optimizing analysis, visualization, and navigation of large image data sets: one 5000-section CT scan can ruin your whole day. Radiology 259:346-362. https://doi.org/10.1148/radiol.11091276

24. Ampanozi G, Zimmermann D, Hatch GM et al (2012) Format preferences of district attorneys for post-mortem medical imaging reports: understandability, cost effectiveness, and suitability for the courtroom: a questionnaire based study. Leg Med (Tokyo) 14:116-120. https://doi.org/10.1016/j.legalmed.2011.12.008

25. Myers B, Hudson SE, Pausch R (2000) Past, present, and future of user interface software tools. ACM Trans Comput Hum Interact 7:3-28. https://doi.org/10.1145/344949.344959

26. Bortolotto C, Eshja E, Peroni C et al (2016) 3D printing of ct dataset: validation of an open source and consumer-available workflow. J Digit Imaging 29:14-21. https://doi.org/10.1007/ s10278-015-9810-8

27. Hodgdon T, Danrad R, Patel MJ et al (2018) Logistics of 3D printing: primer for radiologists. Acad Radiol 25:40-51. https:// doi.org/10.1016/j.acra.2017.08.003

28. Schmuhl H, Heinze O, Bergh B (2013) Use of open source software in health care delivery - results of a qualitative field study. Contribution of the EFMI LIFOSS Working Group. Yearb Med Inform 8:107-113

29. Mildenberger P, Eichelberg M, Martin E (2002) Introduction to the DICOM standard. Eur Radiol 12:920-927. https://doi.org/10. $1007 / \mathrm{s} 003300101100$

30. Hounsfield GN (1980) Computed medical imaging. Med Phys 7:283-290. https://doi.org/10.1118/1.594709

31. Errickson D, Thompson TJU, Rankin BWJ (2014) The application of 3D visualization of osteological trauma for the courtroom: a critical review. J Forensic Radiol Imaging 2:132-137. https://doi. org/10.1016/j.jofri.2014.04.002

32. Kalender WA (2011) Computed tomography: fundamentals, system technology, image quality, applications. John Wiley \& Sons
33. Bharath AA (2008) Introductory medical imaging. Synthesis lectures on biomedical engineering, vol 3, pp 1-186. https://doi.org/ 10.2200/S00165ED1V01Y200811BME026

34. Barrett JF, Keat N (2004) Artifacts in CT: recognition and avoidance. Radiographics 24:1679-1691. https://doi.org/10.1148/rg. 246045065

35. Abdul Rashid SN, Martinez RM, Ampanozi G et al (2013) A rare case of suicide by gunshot with nasal entry assessed by classical autopsy, post-mortem computed tomography (PMCT) and postmortem magnetic resonance imaging (PMMR). J Forensic Radiol Imaging 1:63-67. https://doi.org/10.1016/j.jofri.2013.03.044

36. Brook OR, Eran A, Engel A (2012) CT multiplanar reconstructions (MPR) for shrapnel injury trajectory. Emerg Radiol 19:4351. https://doi.org/10.1007/s10140-011-0988-x

37. Dedouit F, Telmon N, Costagliola R et al (2007) Virtual anthropology and forensic identification: Report of one case. Forensic Sci Int 173:182-187. https://doi.org/10.1016/j.forsciint.2007. 01.002

38. Schweitzer W, Ruder T, Thali M, Ringl H (2015) Skull fractures in post-mortem CT: VRT, flat and skin surface projections in comparison. J Forensic Radiol Imaging 3:214-220. https://doi. org/10.1016/j.jofri.2015.10.006

39. Ringl H, Lazar M, Töpker $M$ et al (2015) The ribs unfolded - a CT visualization algorithm for fast detection of rib fractures: effect on sensitivity and specificity in trauma patients. Eur Radiol 25:1865-1874. https://doi.org/10.1007/ s00330-015-3598-2

40. Tohnak S, Mehnert A, Mahoney M, Crozier S (2009) Dental identification system based on unwrapped CT images. Conf Proc IEEE Eng Med Biol Soc 2009:3549-3552. https://doi.org/ 10.1109/IEMBS.2009.5332483

41. Lundström C, Persson A, Ross S et al (2012) State-of-the-art of visualization in post-mortem imaging. APMIS 120:316-326. https://doi.org/10.1111/j.1600-0463.2011.02857.x

42. Jackowski C, Aghayev E, Sonnenschein M et al (2005) Maximum intensity projection of cranial computed tomography data for dental identification. Int J Legal Med 120:165-167. https:// doi.org/10.1007/s00414-005-0050-1

43. Jackowski C, Lussi A, Classens M et al (2006) Extended CT scale overcomes restoration caused streak artifacts for dental identification in CT-3D color encoded automatic discrimination of dental restorations. J Comput Assist Tomogr 30:510-513

44. Napel S, Rubin GD, Jeffrey RB (1993) STS-MIP: a new reconstruction technique for CT of the chest. J Comput Assist Tomogr $17: 832-838$

45 Brogdon G (2010) Forensic Radiology -, 2nd edn. CRC Press, Boca Raton

46. Remy-Jardin M, Remy J, Gosselin B et al (1996) Sliding thin slab, minimum intensity projection technique in the diagnosis of emphysema: histopathologic-CT correlation. Radiology 200:665-671. https://doi.org/10.1148/radiology.200.3.8756912

47. Levy AD, Harcke HT, Getz JM et al (2007) Virtual autopsy: two- and three-dimensional multidetector CT findings in drowning with autopsy comparison1. Radiology. https://doi.org/10. 1148/radiol.2433061009

48. Thali MJ, Markwalder T, Jackowski C et al (2006) Dental CT imaging as a screening tool for dental profiling: advantages and limitations. J Forensic Sci 51:113-119. https://doi.org/10. 1111/j.1556-4029.2005.00019.x

49. Pommert A, Höhne KH, Pflesser B et al (2001) Ein realistisches dreidimensionales Modell der Inneren Organe auf der Basis des Visible Human. In: Handels H, Horsch A, Lehmann T, Meinzer H-P (eds) Bildverarbeitung für die Medizin 2001. Springer, Berlin, pp 72-75

50. Ebert LC, Schweitzer W, Gascho D et al (2017) Forensic 3D visualization of $\mathrm{CT}$ data using cinematic volume rendering: a 
preliminary study. AJR Am J Roentgenol 208:233-240. https:// doi.org/10.2214/AJR.16.16499

51. Zanola S, Fabrikant SI, Çöltekin A (2009) The effect of realism on the confidence in spatial data quality in stereoscopic 3D displays. In: Proceedings of the 24th International Cartography Conference (ICC 2009), Santiago, Chile, November 15-21

52. Smallman HS, Cook MB (2011) Naïve realism: folk fallacies in the design and use of visual displays. Top Cogn Sci 3:579-608. https://doi.org/10.1111/j.1756-8765.2010.01114.x

53. Visser M, Müller DMJ, van Duijn RJM et al (2019) Inter-rater agreement in glioma segmentations on longitudinal MRI. Neuro Image Clinical 22:101727. https://doi.org/10.1016/j.nicl.2019. 101727

54. Ford JM, Decker SJ (2016) Computed tomography slice thickness and its effects on three-dimensional reconstruction of anatomical structures. J Forensic Radiol Imaging 4:43-46. https:// doi.org/10.1016/j.jofri.2015.10.004

55. Adams R, Bischof L (1994) Seeded region growing. IEEE Trans Pattern Anal Mach Intell 16:641-647. https://doi.org/10.1109/34. 295913

56. Smistad E, Falch TL, Bozorgi M et al (2015) Medical image segmentation on GPUs-a comprehensive review. Med Image Anal 20:1-18. https://doi.org/10.1016/j.media.2014.10.012

57 Pham DL, Xu C, Prince JL (2000) Current methods in medical image segmentation. Annu Rev Biomed Eng 2:315-337. https:// doi.org/10.1146/annurev.bioeng.2.1.315

58. Pal NR, Pal SK (1993) A review on image segmentation techniques. Pattern Recogn 26:1277-1294. https://doi.org/10.1016/ 0031-3203(93)90135-J

59. Haralick RM, Shapiro LG (1985) Image segmentation techniques. Comput Vis Graph Image Process 29:100-132. https://doi.org/10. 1016/S0734-189X(85)90153-7

60. Mortensen EN, Barrett WA (1995) Intelligent scissors for image composition. In: Proceedings of the 22 nd annual conference on Computer graphics and interactive techniques - SIGGRAPH '95. ACM Press, New York, p 191-198

61. Roth HR, Shen C, Oda H et al (2018) Deep learning and its application to medical image segmentation 36:9

62. Wachinger C, Sharp GC, Golland P (2013) Contour-driven regression for label inference in atlas-based segmentation. Med Image Comput Comput Assist Interv 16:211-218. https://doi.org/10. 1007/978-3-642-40760-4 27

63. Hoogendoorn C, Duchateau N, Sánchez-Quintana D et al (2013) A high-resolution atlas and statistical model of the human heart from multislice CT. IEEE Trans Med Imaging 32:28-44. https:// doi.org/10.1109/TMI.2012.2230015

64. Zhang L, Hoffman EA, Reinhardt JM (2006) Atlas-driven lung lobe segmentation in volumetric X-ray CT images. IEEE Trans Med Imaging 25:1-16. https://doi.org/10.1109/TMI.2005.859209

65. Heimann T, Meinzer H-P (2009) Statistical shape models for 3D medical image segmentation: A review. Med Image Anal 13:543563. https://doi.org/10.1016/j.media.2009.05.004

66. Lorensen WE, Cline HE (1987) Marching cubes: a high resolution $3 \mathrm{~d}$ surface construction algorithm. In: Proceedings of the 14th Annual Conference on Computer Graphics and Interactive Techniques. ACM, New York, p 163-169. https://doi.org/10.1145/ 37401.37422

67. Uldin T (2017) Virtual anthropology - a brief review of the literature and history of computed tomography. Forensic Sci Res 2:165-173. https://doi.org/10.1080/20961790.2017.1369621

68. Raul J-S, Deck C, Willinger R, Ludes B (2008) Finite-element models of the human head and their applications in forensic practice. Int J Legal Med 122:359-366. https://doi.org/10.1007/ s00414-008-0248-0

69. Ebert LC, Thali MJ, Ross S (2011) Getting in touch-3D printing in forensic imaging. Forensic Sci Int 211:e1-6. https://doi.org/10. 1016/j.forsciint.2011.04.022

70. Kottner S, Flach PM, Gascho D et al (2019) Communicating 3D data-interactive 3D PDF documents for expert reports and scientific publications in the field of forensic medicine. Int J Legal Med. https://doi.org/10.1007/s00414-019-02156-4

71. Schweitzer W, Thali M (2015) Computed tomography of scalp embedded gravel: Differentiation between falling and kicking. J Forensic Radiol Imaging 3:200-209. https://doi.org/10.1016/j. jofri.2015.09.002

72. Ballard DH, Trace AP, Ali S et al (2018) Clinical applications of 3d printing: primer for radiologists. Acad Radiol 25:52-65. https://doi.org/10.1016/j.acra.2017.08.004

73. Schweitzer W, Thali M, Aldomar E, Ebert L (2020) Overview of the use of $3 \mathrm{D}$ printing in forensic medicine. Rechtsmedizin 30:292-299. https://doi.org/10.1007/s00194-020-00412-1

74. Carew RM, Errickson D (2020) An overview of 3D printing in forensic science: the tangible third-dimension. J Forensic Sci 65:1752-1760. https://doi.org/10.1111/1556-4029.14442

75. Decker S, Ford J, Davy-Jow S et al (2013) Who is this person? A comparison study of current three-dimensional facial approximation methods. Forensic Sci Int 229:161.e1-8. https://doi.org/10. 1016/j.forsciint.2013.03.028

76. Woźniak K, Rzepecka-Woźniak E, Moskała A et al (2012) Weapon identification using antemortem computed tomography with virtual 3D and rapid prototype modeling-a report in a case of blunt force head injury. Forensic Sci Int 222:e29-e32. https:// doi.org/10.1016/j.forsciint.2012.06.012

77. Baier W, Warnett JM, Payne M, Williams MA (2018) Introducing 3D printed models as demonstrative evidence at criminal trials. J Forensic Sci 63:1298-1302. https://doi.org/10.1111/1556-4029. 13700

78. Carew RM, Morgan RM, Rando C (2019) A preliminary investigation into the accuracy of 3D modeling and 3D printing in forensic anthropology evidence reconstruction. J Forensic Sci 64:342-352. https://doi.org/10.1111/1556-4029.13917

79. Baier W, Norman DG, Donnelly MJ, Williams MA (2020) Forensic 3D printing from micro-CT for court use- process validation. Forensic Sci Int 318:110560. https://doi.org/10.1016/j.forsciint. 2020.110560

80. Stanco F, Battiato S, Gallo G (2011) Digital imaging for cultural heritage preservation: analysis, restoration, and reconstruction of ancient artworks. CRC Press, Boca Raton

81. Kruth JP (1991) Material incress manufacturing by rapid prototyping techniques. CIRP Ann 40:603-614. https://doi.org/10.1016/ S0007-8506(07)61136-6

82. Schweitzer W, Flach PM, Thali M et al (2016) Very economical immersion pump feasibility for postmortem CT angiography. J Forensic Radiol Imaging 5:8-14. https://doi.org/10.1016/j.jofri. 2015.11.009

83. Beurden MHPH van, IJsselsteijn WA, Juola JF (2012) Effectiveness of stereoscopic displays in medicine: a review. 3D Res 3:1-13. https://doi.org/10.1007/3DRes.01(2012)3

84. Kilgus T, Heim E, Haase $\mathrm{S}$ et al (2015) Mobile markerless augmented reality and its application in forensic medicine. Int $\mathrm{J}$ CARS 10:573-586. https://doi.org/10.1007/s11548-014-1106-9

85. Affolter R, Eggert S, Sieberth T et al (2019) Applying augmented reality during a forensic autopsy-Microsoft HoloLens as a 
DICOM viewer. J Forensic Radiol Imaging 16:5-8. https://doi. org/10.1016/j.jofri.2018.11.003

86. Arikatla S, Fillion-Robin J-C, Paniagua B, et al (2018) Bringing virtual reality to 3D slicer. In: Kitware Blog. https://blog.kitware. com/slicervirtualreality/. Accessed 1 Apr 2020

87. Ebert LC, Nguyen TT, Breitbeck R et al (2014) The forensic holodeck: an immersive display for forensic crime scene reconstructions. Forensic Sci Med Pathol 10:623-626. https://doi.org/10. 1007/s12024-014-9605-0

88. Sieberth T, Dobay A, Affolter R, Ebert L (2019) A toolbox for the rapid prototyping of crime scene reconstructions in virtual reality.
Forensic Sci Int 305:110006. https://doi.org/10.1016/j.forsciint. 2019.110006

89. Sieberth T, Dobay A, Affolter R, Ebert LC (2019) Applying virtual reality in forensics - a virtual scene walkthrough. Forensic Sci Med Pathol 15:41-47. https://doi.org/10.1007/s12024-018-0058-8

Publisher's Note Springer Nature remains neutral with regard to jurisdictional claims in published maps and institutional affiliations. 